\title{
From the Executive Editor
}

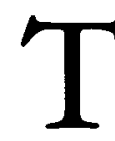

His edition of CARDIOLOGY IN THE Young IS full, as ever, with articles on a wide spectrum of cardiac disease in the young. We are grateful to our authors for the submission of an increasing number of good quality manuscripts. With limitations of space, that inevitably means that we are rejecting a higher proportion of manuscript we receive, but we believe that the journal is getting ever stronger. Do you agree? We welcome feedback from our readers. Are there any subjects that you think we should cover that we do not? Do you disagree with the conclusions of any studies we publish? Let us know. We will take note of any comments we receive, and we will publish well-argued or interesting letters.

We aim to ensure each edition of the journal is well balanced. Here we have included a major review of Marfan's disease, together with an article forming part of our series on continuing medical education, this time addressing that most complex of cardiac anomalies, tetralogy of Fallot with pulmonary atresia. We hope you continue to find these reviews interesting and useful, but it is the original articles that form the backbone of any scientific journal. Here, we have included a diverse selection. There are articles on interventional procedures, surgical outcomes, and imaging techniques. We are particularly keen to publish more articles on the surgical repair of congenital cardiac anomalies, and on paediatric cardiac intensive care. We welcome the submission of high quality articles in these areas, but we are interested in any subjects related to the diagnosis, treatment, or followup of heart disease in children.

The readability and appearance of the journal is important. This is why we have always sought to publish well-illustrated articles. We have always published colour figures as a matter of course, which is not the case in other journals. In this issue, we are continuing our series "Images in Congenital Heart Disease". We welcome the submission of interesting images of high quality for publication. We are sent a great number of brief reports, of which we can only publish very few. Authors wishing to submit such reports should read our instructions to authors carefully. We have to be very selective, and only reports that we and our reviewers find particularly original or interesting will be published.

One report that we have decided to publish is the description by Sebening and colleagues (pp 414-416) describing myocardial bridging in a child with hypertrophic cardiomyopathy. The authors provided excellent quality video loops of the angiograms and surgical findings in this patient. We have included these on our website bttp://www.greenwich-medical.co.uk so that readers can have an opportunity to review them. We will be pleased to publish video loops on the website to accompany articles in future issues. Subscribers now have access to the full journal, and everyone can contribute to the Forum for Discussion at the website. Sebening and colleagues have asked for responses to their article to be posted there. We will consider publishing any responses in future issues of the journal.

Our journal is what you, our readers and authors, make it. Your contribution, original articles, or feedback will help us build on its success. We look forward to your response to this case report, or any other comments on the journal, either by letter or to our internet discussion forum.

\author{
Dr Edward Baker \\ Department of Paediatric Cardiology, \\ Guy's Hospital, St Thomas Street, \\ London SE1 9RT, UK \\ E-mail:Edward.baker@kcl.ac.uk
}

